\title{
Abuse potential of propofol used for sedation in gastric endoscopy and its correlation with subject characteristics
}

\author{
Ja Hyun Kim ${ }^{1}$, Heewon Byun ${ }^{2}$, and Jun Hyun Kim ${ }^{3}$ \\ ${ }^{1}$ Department of Occupational \& Environmental Medicine, Samsung Changwon Hospital, Sungkyunkwan University School of \\ Medicine, ${ }^{2}$ Department of Health Promotion Center, Samsung Changwon Hospital, Sungkyunkwan University School of Medicine, \\ Changwon, ${ }^{3}$ Department of Anesthesiology and Pain Medicine, Ilsan Paik Hospital, Inje University School of Medicine, Goyang, Korea
}

Background: Propofol has been widely used for an induction and/or maintenance of general anesthesia, or for sedation for various procedures. Although it has many ideal aspects, there have been several cases of drug abuse and addiction. The authors investigated whether there are abuse liable groups among the general population.

Methods: We surveyed 169 patients after gastric endoscopic examination, which used propofol as a sedative, with the Addiction Research Center Inventory (ARCI) questionnaire. Other characteristics of the patients, such as past history, smoking habits, depression, anxiety, alcohol abuse liability and sleep disturbance, were recorded by history taking and several questionnaires before the exam.

Results: Propofol had a high Morphine-Benzedrine Group (MBG) score (representative value for euphoria) of 6.3, which is higher than marijuana, and a Pentobarbital-Chlorpromazine-Alcohol Group (PCAG) score (representative value of sedation) of 8.1, which is lower than most opioids. The MBG score showed no statistically significant correlation between any of the characteristics of the groups. In females, the PCAG score showed a correlation with age, and in males, it showed a correlation with a sleeping problem.

Conclusions: Propofol had relatively high euphoria and low residual sedative effects. It had a more potent sedative effect in the female group who were young, and in the male group who had a low sleep quality index. There were differences in the abuse liability from a single exposure to propofol in the general population. Further study is needed to evaluate the abuse liability of repeated exposure. (Korean J Anesthesiol 2013; 65: 403-409)

Key Words: Addictive behavior, Dependency, Hypnotics and sedatives, Intravenous substance abuse, Propofol.

\footnotetext{
Received: March 22, 2013. Revised: May 3, 2013. Accepted: May 28, 2013.

Corresponding author: Jun Hyun Kim, M.D., Department of Anesthesiology and Pain Medicine, Ilsan Paik Hospital, Inje University School of Medicine, 2240, Daewha-dong, Ilsanseo-gu, Goyang 411-706, Korea. Tel: 82-31-910-7160, Fax: 82-31-910-7184, E-mail: urdesire@hanmail.net (c) This is an open-access article distributed under the terms of the Creative Commons Attribution Non-Commercial License (http:// creativecommons.org/licenses/by-nc/3.0/), which permits unrestricted non-commercial use, distribution, and reproduction in any medium, provided the original work is properly cited.
} 


\section{Introduction}

Propofol is an intravenous anesthetic widely used in the induction of anesthesia and sedation and in various procedures. This drug has desirable characteristics such as prompt anesthesia induction and recovery, but due to several current cases of abuse, Korea was first in the world to designate propofol as a psychoactive drug on February 1, 2011. Despite such attempts to legally regulate the use of propofol, there still have been reports of several cases of abuse. However, although there are legal regulations and procedures to report abuse, the authors do not know of any precedent research regarding the degree of addiction caused by propofol in the general population, and whether there is a group particularly vulnerable to its addictiveness. Through a subjective report conducted on people who received propofol during an endoscopy for a medical check-up, the authors examined the degree of abuse liability for propofol in the general population, and investigated whether there was a group with a particularly higher abuse liability and examined the characteristics of that group.

\section{Materials and Methods}

This study was conducted with the approval of the Institutional Review Board of the hospital. It was conducted on people who received conscious sedation endoscopy induced by propofol in a single general hospital from June to August of 2012. One hundred eighty-five people were selected as research subjects during this period, and from these, the study was conducted on 169 who agreed to participate in the survey and study. There were 88 males and 81 females, aged from 25 to 70 . The mean age of males and its standard deviation were $50.9 \pm 7.1$, and 41.9 \pm 7.0 for females, and there was no significant difference in age between sexes ( $\mathrm{P}=0.99$, Table 1$)$.

The subjects were people with no allergies or any particular reactions to medication, and were people who answered on the survey conducted before the examination that they had no history of mental illness, or had received psychiatric treatment for psychiatric disorders such as depression. The study was done on people who had not been administered propofol previously, or had no memory of being administered propofol.

Basic medical history and past history were included in the survey conducted before the examination, and smoking habits, drinking problem, depression tendency, anxiety tendency, and sleep status were evaluated using a structured questionnaire to examine the relevance to the Morphine-Benzedrine Group (MBG) score, the Pentobarbital-Chlorpromazine-Alcohol Group (PCAG) score of the Addiction Research Center Inventory (ARCI) $[1,2]$, and street value.

The Alcohol Use Disorders Identification Test (AUDIT) was used to evaluate drinking problem. This questionnaire, composed of 10 questions, was developed by the World Health Organization to differentiate persons with problematic drinking behaviors before the alcohol dependence stage is reached, while reflecting quantitative aspects of drinking. It is currently the most widely used among the screening tests utilized to evaluate drinking problem. It is expressed as a score of $0-40$ and a higher score reflects a higher risk. A score of 12 or more indicates a habitual drinker who needs caution; 15 or more is a problematic drinker who needs appropriate intervention, and 25 or more is an alcoholic who needs professional hospital treatment and counseling [3]. The Beck Anxiety Inventory (BAI) was used to evaluate anxiety tendency. It consists of 21 questions, was developed to differentiate anxiety from depression, and includes the cognitive, emotional, and physical aspects of anxiety. The score is expressed as 0-63, and 0-22 is interpreted as normal, 22-26 as a state of anxiety (requires observation and intervention), 27-31 as a state of serious anxiety, and 32 or more as a state of intense anxiety [4]. The Center for Epidemiologic Studies Depression (CES-D) scale questionnaire was used to measure depression tendency. This consists of 20 questions. It is a primary differentiation tool for depression which measures the degree of depression based on the duration period of the symptoms. It was developed in 1977 and used in epidemiologic research. This study used the Korean version [5]. The score is expressed as 0-60, and the degree of depression is more severe as the score becomes higher [6]. The Pittsburgh Sleep Quality Index (PSQI) was used for sleep status. It consists of 19 questions, and shows the sleeping habits and various aspects of sleep disorder during the last month. It evaluates and gives a score to 7 areas; subjective sleep quality, sleep latency, sleep duration, habitual sleep effect, sleep disturbance, use of sleeping pills and daytime dysfunction. The score is expressed as $0-21$, and a score of 6 or more signifies that the quality of sleep is bad [7].

The total amount of propofol $(2 \mathrm{mg} / \mathrm{kg})$ used in the examination was divided and IV administered for the convenience of the procedure. While performing the endoscopy, the heart rate, blood pressure and oxygen saturation were monitored using ECG, automated non invasive blood pressure monitor and pulse oximetry.

After completing the endoscopy, the subjects were moved to the recovery room, and the survey was conducted within $30 \mathrm{~min}$ utes of recovery. Time taken to complete the survey was generally within 5 minutes.

The survey consisted of a total of 33 questions using the MBG scale, PCAG scale provided by ARCI, and street value. Questions $1-17$ of the survey covered the MBG scale and 18-32 the PCAG scale. Questions 29-32 of the PCAG scale are false questions where 1 point is given to 'no', and 0 points is given to 'yes'. The MBG scale assesses euphoria and is expressed as a score of 
$0-17$, and the PCAG scale assesses sedation and is expressed as a score of $0-15$. Street value is a question whether patients will buy this medication and how much they are willing to pay for it (Appendix 1).

In addition to the survey, the subjects underwent a physical examination that included height, weight and blood pressure and blood tests such as complete blood count, liver function test, Lipid profile and thyroid function test.

\section{Statistics}

A mean comparison was performed on the survey responses to see if there were any differences in the basic characteristics of the subjects such as age or sex, and a correlation analysis was performed to see whether age, sex, weight, liver enzyme values, and blood pressure were related to the MBG and PCAG scores. Multiple regression analysis was performed on the results for age, smoking habits, drinking problem, depression tendency, anxiety tendency and sleep status with the MBG and PCAG scores according to sex. Based on the results of the regression analysis, the categories with a low regression coefficient were consecutively removed to perform a backward multiple regression analysis to find the most appropriate model. The analysis results were considered to be significant when the $\mathrm{P}$ value was less than 0.05. The software package R (3.0; Copyright (C) 2013 The R Foundation for Statistical Computing) was used for the statistical analysis.

\section{Results}

In the survey conducted within 30 minutes of recovery, the mean MBG score was 6.3; the mean PCAG score was 8.1, and there were no differences according to sex (Table 1). The results of the correlation analysis showed that there were no associated elements for the MBG score, but the PCAG score decreased as the age increased (correlation coefficient $-0.241, \mathrm{P}=0.002$ ). Sixty-seven out of 169 did not respond to the question on street value; 35 responded they would not pay, and 67 responded that they would pay to purchase propofol. The mean street value of the respondents was $38,000 \mathrm{KRW}$, and the mean price for respondents who were willing to pay was 58,000 KRW. The street value did not show a difference according to age or sex (Table 1). The results of the correlation analysis showed that street value tended to be higher when the MBG score was higher, but it was not statistically significant $(\mathrm{P}=0.20, \mathrm{r}=0.127)$. The PCAG score did not show a correlation with street value.

The authors examined the relationship between the MBG and PCAG scores and personal factors and the survey results of the subjects. The weight, blood pressure, thyroid function test results, and liver enzyme values did not show any significant relationship to the scores. A correlation analysis was performed on each score with age, smoking, drinking, depression, anxiety and sleep. When male and female results were observed together, there were no factors which showed a correlation with the MBG or PCAG score. However, when sex was differentiated in the

Table 1. General Features

\begin{tabular}{|c|c|c|c|c|}
\hline & Total $(\mathrm{n}=169)$ & Male $(\mathrm{n}=88)$ & Female $(\mathrm{n}=81)$ & $P$ value \\
\hline Age (yr) & $42.9 \pm 7.1$ & $50.9 \pm 7.1$ & $41.9 \pm 7.0$ & 0.99 \\
\hline Weight (kg) & $64.6 \pm 11.9$ & $72.2 \pm 10.4$ & $56.2 \pm 6.7$ & 0.00 \\
\hline Height (cm) & $165.5 \pm 8.4$ & $171.6 \pm 5.7$ & $158.8 \pm 5.1$ & 0.00 \\
\hline MBG score (0-17) & $6.3 \pm 5.7$ & $6.6 \pm 5.9$ & $6.1 \pm 5.4$ & 0.60 \\
\hline PCAG score $(0-15)$ & $8.1 \pm 3.3$ & $7.8 \pm 3.4$ & $8.4 \pm 3.2$ & 0.20 \\
\hline Street value $\left(10 \mathrm{k} K \mathrm{KWW}^{*}\right)$ & $3.8 \pm 12.6(n=102)$ & $4.1 \pm 13.6(n=54)$ & $3.5 \pm 11.5(\mathrm{n}=48)$ & 0.79 \\
\hline
\end{tabular}

Values are expressed as means \pm SD. MBG: Morphine-Benzedrine Group, PCAG: Pentobarbital-Chlorpromazine-Alcohol Group. *Korean Won.

Table 2. Multiple Linear Regression of Factors with MBG Score

\begin{tabular}{|c|c|c|c|c|c|c|}
\hline & \multicolumn{2}{|c|}{ Estimate coefficient } & \multicolumn{2}{|c|}{ Standard error } & \multicolumn{2}{|c|}{ P value } \\
\hline & Male & Female & Male & Female & Male & Female \\
\hline Age & 0.092 & 0.129 & 0.137 & 0.109 & 0.507 & 0.243 \\
\hline Smoking & 1.331 & NA & 1.587 & NA & 0.406 & NA \\
\hline AUDIT $(0-40)$ & -0.091 & -0.162 & 0.149 & 0.332 & 0.545 & 0.628 \\
\hline BAI $(0-63)$ & 0.072 & -0.142 & 0.188 & 0.211 & 0.701 & 0.504 \\
\hline PSQI (0-21) & -0.020 & -0.242 & 0.313 & 0.292 & 0.950 & 0.413 \\
\hline CES-D (0-60) & 0.245 & 0.022 & 0.197 & 0.147 & 0.219 & 0.882 \\
\hline
\end{tabular}

Smoking status (smoking, nonsmoking), Alcohol (AUDIT score), Anxiety (BAI score), Sleeping problem (PSQI score), Depression (CES-D score). MBG: Morphine-Benzedrine Group, AUDIT: Alcohol Use Disorders Identification Test, BAI: Beck Anxiety Inventory, PSQI: Pittsburgh Sleep Quality Index, CES-D: Center for Epidemiologic Studies Depression. 
Table 3. Multiple Linear Regression of Factors with PCAG Score

\begin{tabular}{|c|c|c|c|c|c|c|}
\hline & \multicolumn{2}{|c|}{ Estimate coefficient } & \multicolumn{2}{|c|}{ Standard error } & \multicolumn{2}{|c|}{$P$ value } \\
\hline & Male & Female & Male & Female & Male & Female \\
\hline Age & -0.076 & -0.194 & 0.076 & 0.058 & 0.319 & $0.002 *$ \\
\hline Smoking & -1.540 & NA & 0.877 & NA & 0.805 & NA \\
\hline AUDIT $(0-40)$ & -0.069 & -0.308 & 0.082 & 0.176 & 0.409 & 0.086 \\
\hline BAI $(0-63)$ & -0.111 & 0.181 & 0.104 & 0.112 & 0.289 & 0.112 \\
\hline PSQI (0-21) & 0.451 & -0.154 & 0.173 & 0.154 & $0.012 *$ & 0.324 \\
\hline CES-D (0-60) & 0.034 & 0.007 & 0.109 & 0.078 & 0.757 & 0.924 \\
\hline
\end{tabular}

Smoking status (smoking, nonsmoking), Alcohol (AUDIT score), Anxiety (BAI score), Sleeping problem (PSQI score), Depression (CES-D score). *P value < 0.05. PCAG: Pentobarbital-Chlorpromazine-Alcohol Group, AUDIT: Alcohol Use Disorders Identification Test, BAI: Beck Anxiety Inventory, PSQI: Pittsburgh Sleep Quality Index, CES-D: Center for Epidemiologic Studies Depression.

Table 4. Ten Largest Data of Street Value, and Corresponding MBG Score, PCAG Score

\begin{tabular}{cccc}
\hline Street value $\left(10 \mathrm{kRW}^{*}\right)$ & Gender & MBG score & PCAG score \\
\hline 100 & M & 13 & 7 \\
80 & $\mathrm{~F}$ & 5 & 7 \\
10 & $\mathrm{~F}$ & 17 & 11 \\
10 & $\mathrm{M}$ & 17 & 11 \\
10 & $\mathrm{M}$ & 10 & 8 \\
10 & $\mathrm{M}$ & 9 & 9 \\
10 & $\mathrm{M}$ & 6 & 12 \\
10 & $\mathrm{M}$ & 5 & 8 \\
5 & $\mathrm{M}$ & 17 & 6 \\
5 & $\mathrm{M}$ & 17 & 5 \\
Average: 25 & M: 8, F: 2 & Average: 11.6 & Average: 8.4 \\
\hline
\end{tabular}

MBG: Morphine-Benzedrine Group, PCAG: Pentobarbital-Chlorpromazine-Alcohol group, M: male, F: Female. *Korean Won.

analysis, the PCAG score showed a significant correlation with age in women while the PCAG score showed a significant correlation with sleep status in men (Tables 2 and 3 ).

For street value, there were outliers that made the standard deviation to be more than 3 times the mean (Table 1). To examine such outliers or values which widely deviated from the mean, the MBG and PCAG scores of the upper 10 street value responses are presented in Table 4.

\section{Discussion}

Propofol is a drug which works on the gamma-aminobutyric acid $_{\mathrm{A}}$ receptor, and alcohol, barbiturates, and benzodiazepine also work on this receptor. In addition, propofol activates the mesocorticolimbic dopaminergic circuit which is thought to be a major mechanism for addiction [8]. Even in research conducted on a small number of healthy volunteers, propofol was shown with certainty to function as a reward [9]. However, the effect of the drug quickly disappears when no longer administered, and severe respiration suppression occurs with an increase in the concentration; thus, cases of abuse are not largely known compared to other drugs. The reported cases of abuse were mostly medical personnel who had easy access to the drug. However, recently, there have been reports of abuse by patients in medical institutes, and even after the drug was categorized as psychoactive drug, there have been reports of abuse by people other than medical personnel [10]. When this drug is abused, it is more problematic than other drugs because not only is there a dependence on the drug, but it can easily lead to death due to respiratory suppression which can be caused by the drug itself.

Propofol is not used by itself as a treatment modality, so it is inevitably connected to various procedures. Currently, this drug is mostly widely used as an inducing agent for general anesthesia. There have been reports that elation was felt when awakening from general anesthesia after receiving propofol, but general anesthesia is connected with unpleasant memories such as surgery and postoperative pain; therefore, it is difficult to evaluate the addictiveness or abuse liability of propofol itself [11]. Hence, the authors performed an investigation on patients receiving an endoscopic examination which has less or no pain after the procedure.

ARCI is a questionnaire consisting of 550 items, and it was developed in 1966 to examine the physical, emotive, cognitive, and subjective effects of drugs. In 1971, Martin et al. developed a short form consisting of 49 items which has 5 scales - MBG measuring euphoria, PCAG measuring sedation, lysergic acid diethylamide group measuring dysphoria, Benzedrine group relevant to stimulant-sensitive scale, and $\mathrm{A}$ which measures the effect of d-amphetamine [12].

It is not accurately known which of the feelings caused by propofol leads to addiction, but according to the media or precedent reports, sleep inducement and euphoria are commonly mentioned together $[9,11]$. For this reason, the authors chose MBG and PCAG from the ARCI inventory as survey items for subjective effect.

Compared to the MBG score of 4.7 and PCAG score of 5.9 calculated from a precedent study on marijuana, the MBG and PCAG scores in our study were 6.3 and 8.1 , which indicate high- 
er levels in both euphoria and sedation [13]. In addition, in another study conducted with morphine $10 \mathrm{mg}$ and buprenorphine $0.3 \mathrm{mg}$, the PCAG scores were 9.8 and 11.4, respectively, which is higher than the PCAG score of 8.1 for propofol in this study [14]. In this study conducted on opioids, the PCAG values were measured in 60 minute intervals up to 300 minutes after administering the opioids, and the maximum value appeared at around 60 minutes. Considering that the PCAG in our study was measured around 30 minutes after stopping the propofol, it could show that the sedative effect of propofol quickly disappears once administration is stopped. In addition, there were many people who gave a higher street value because the MBG score for euphoria was higher, but this was not statistically significant. On the other hand, people who had a higher PCAG score for sedation responded with a lower street value, which suggests that the sleep inducing effect of propofol may have less effect on abuse liability in the general population. When precedent studies conducted on benzodiazepines were examined, after administering the drug, the PCAG score increased but the MBG score did not increase in non-drug abusing volunteers. Additionally, when tested on drug abusers, there were cases in which the liking rate or street value increased after administering the relevant drug but the MBG did not increase; thus, it has been reported that the MBG score may be less sensitive than other two scales [2]. Therefore, it is unusual that the MBG score appears to be high in our study, which was conducted on the general population.

There are various results in precedent studies regarding the effect of PCAG on the preference of drugs. PCAG had a correlation or did not have a correlation to MBG or other liking rates according to the drug description and whether the subjects were general population or people with a past history of drug or alcohol abuse [2]. This suggests that the preference for the sedative effect of a drug differs according to individuals. Therefore, it is considered that the sleep inducing effect mentioned by the media or addicts may have less weight in deciding the preference for propofol than what has been reported.

This study measured the preference for propofol after a single incident of exposure. Therefore, there is need for further study regarding which characteristics cause abuse when repeatedly exposed to the drug. In single exposure, such as our study, the euphoria caused by propofol was a larger factor for drug preference rather than the sedative effect, and the euphoric effect of propofol measured in our study was also higher than other drugs. The sedative effect of propofol such as sleep inducement disappears quickly and has less remaining effect compared to other drugs such as opioids; thus, it has less influence on the mechanism for preference.

The comparison results for depression tendency, anxiety tendency, sleep status and drinking problem obtained from the survey with the MBG and PCAG scores showed that age was related to the PCAG score in women, while sleep status was related to the PCAG score in men.

The PCAG score showed a significant correlation where it decreased as age increased in females. It is unusual that the sedative effect of the drug was felt less by the older population despite the strong possibility of a higher blood concentration of propofol at the time of survey because of a decreased metabolic rate of the drug depending on age. However, the size of the correlation coefficient is small thus its significance must be interpreted with caution. In addition, the PCAG score tended to be high in groups with a low risk of drinking problems, although this was not statistically significant. It can be inferred that the sedative effect appears larger when there is less preference for alcohol. However, there was a report showing that the required dosage of propofol for inducing anesthesia increases for alcoholics who drink a large amount of alcohol [15]. Taking this point into consideration, the higher PCAG figures in the low drinking hazard group could simply be caused by the same amount of propofol having a larger sedative effect because the group had less exposure to alcohol in the past. There is controversy whether the group with the higher PCAG score had higher abuse liability. For euphoria, it is obvious that higher figures will increase the risk of drug preference, but for the sedative effect, some people will prefer the feeling while some will not. In studies regarding various sedatives, the relationship between the PCAG score and liking rate showed different results based on whether the subjects were drug abusers or general population, and what kind of drug was used [2]. However, if a certain drug has a large sedative effect, there is a higher possibility of leading people with abuse liability to addiction compared to other drugs with no or less sedative effect. Taken together, it is difficult to interpret this high PCAG score group as a group with a particularly higher possibility of propofol addiction.

For men, the PCAG had a significant correlation with PSQI survey score evaluating sleep status, and this can be seen as the sedative effect of propofol appearing larger in males with bad sleep status. In a study conducted on people with insomnia, it was reported that the subjects reached a deeper state of sleep when IV injected with propofol; thus, it can be inferred that the population with sleep debt reached a deeper sleep state than the general population when administered propofol and consequently, felt a larger sedative effect after stopping the administration of propofol [16].

The non-response rate for street value was high (39.6\%) so it can be seen as having less statistical effectiveness. However, despite the overall mean of $38,000 \mathrm{KRW}$, the upper 10 values ranged from 1,000,000 KRW to 50,000 KRW and their mean was $250,000 \mathrm{KRW}$ which is a very high price range. When these upper 10 values were excluded, the mean street value was only $16,900 \mathrm{KRW}$. The mean MBG score of these 10 subjects was also 
11.6, which is considerably higher than the overall mean of 6.3 . The mean PCAG score of these 10 subjects did not show a great difference with the overall mean. This suggests that when street value is considered as the liking rate, there is a group which shows a large preference value compared to the mean preference, and this group is more likely to have higher MBG scores.

The limitation of this study is that it was based on a selfadministered questionnaire. It is difficult for researchers to accurately evaluate the tendency for addiction, which is fairly influenced by the subjective preference for a drug. Hence, the only method of determining the type of reward a drug causes is to ask each individual to report subjective feelings, and there are limits to objectifying this response into scores, as such scores may skew the real effect. However, this research method was used to evaluate the abuse liability of various drugs since the development of the ARCI questionnaire [2]. For street value, the response rate noticeably declined compared to other questions, and this may be because the subjects avoided responding because they misunderstood it as a question asking whether they would pay for additional cost when there is further testing.
The significance of this study is that it evaluated the abuse liability of a drug used in common procedures on the general population with a structured survey. The study results showed that there were groups with different MBG and PCAG scores within the general population so there could be a group more vulnerable to propofol addiction, and that the degree of euphoria from propofol can be considerably large even in the general population. In addition, euphoria is more likely to influence the liking of the drug from a single exposure. Moreover, for the aspect of sedation, the PCAG score of propofol was lower than that of other drugs so factors related to sleep such as 'refreshing sleep' and 'sensation of good rest' mentioned by addicts had less influence compared to other drugs. A population group who felt particularly higher euphoria from propofol was not differentiated. The female group with younger age and male group with bad sleep status felt the sedative effect of propofol stronger than other population groups. There is need for additional research regarding which characteristics of propofol cause addiction when there is repeated exposure.

\section{References}

1. Haertzen CA, Hill HE, Belleville RE. Development of the addiction research center inventory (ARCI): selection of items that are sensitive to the effects of various drugs. Psychopharmacologia 1963; 4: 155-66.

2. de Wit H, Griffiths RR. Testing the abuse liability of anxiolytic and hypnotic drugs in humans. Drug Alcohol Depend 1991; $28: 83-111$.

3. Saunders JB, Aasland OG, Babor TF, de la Fuente JR, Grant M. Development of the Alcohol Use Disorders Identification Test (AUDIT): WHO Collaborative Project on Early Detection of Persons with Harmful Alcohol Consumption-II. Addiction 1993; 88: 791-804.

4. Beck AT, Epstein N, Brown G, Steer RA. An inventory for measuring clinical anxiety: psychometric properties. J Consult Clin Psychol 1988; 56: 893-7.

5. Cho MJ, Kim KH. Diagnostic validity of the CES-D (Korean version) in the assessment of DSM-III-R major depression. J Korean Neuropsychiatr Assoc 1993; 32: 381-99.

6. Park JH, Kim KW. A review of the epidemiology of depression in Korea. J Korean Med Assoc 2011; 54: 362-9.

7. Buysse DJ, Reynolds CF 3rd, Monk TH, Berman SR, Kupfer DJ. The Pittsburgh Sleep Quality Index: a new instrument for psychiatric practice and research. Psychiatry Res 1989; 28: 193-213.

8. Pain L, Gobaille S, Schleef C, Aunis D, Oberling P. In vivo dopamine measurements in the nucleus accumbens after nonanesthetic and anesthetic doses of propofol in rats. Anesth Analg 2002; 95: 915-9.

9. Zacny JP, Lichtor JL, Thompson W, Apfelbaum JL. Propofol at a subanesthetic dose may have abuse potential in healthy volunteers. Anesth Analg 1993; 77: 544-52.

10. Park JH, Kim HJ, Seo JS. Medicolegal review of deaths related to propofol administration: analysis of 36 autopsied cases. Korean J Leg Med 2012; 36: 56-62.

11. Koopmann A, von der Goltz C, Hermann D, Kiefer F. Propofol addiction initiated by anesthetic use. Am J Psychiatry 2011; 168: 211-2.

12. Martin WR, Sloan JW, Sapira JD, Jasinski DR. Physiologic, subjective, and behavioral effects of amphetamine, methamphetamine, ephedrine, phenmetrazine, and methylphenidate in man. Clin Pharmacol Ther 1971; 12: 245-58.

13. Greenwald MK, Stitzer ML. Antinociceptive, subjective and behavioral effects of smoked marijuana in humans. Drug Alcohol Depend 2000; 59: 261-75.

14. Zacny JP, Conley K, Galinkin J. Comparing the subjective, psychomotor and physiological effects of intravenous buprenorphine and morphine in healthy volunteers. J Pharmacol Exp Ther 1997; 282: 1187-97.

15. Fassoulaki A, Farinotti R, Servin F, Desmonts JM. Chronic alcoholism increases the induction dose of propofol in humans. Anesth Analg 1993; 77: 553-6.

16. Xu Z, Jiang X, Li W, Gao D, Li X, Liu J. Propofol-induced sleep: efficacy and safety in patients with refractory chronic primary insomnia. Cell Biochem Biophys 2011; 60: 161-6. 


\section{Appendix 1. ARCI Questionnaires and Street Value Question Used for This Study}

-Following questions are about feelings after conscious sedation endoscopy. Please check yes or no.

1. I would be happy all the time if I felt as I feel now.

2. I am in the mood to talk about the feeling I have.

Yes

No $\square$

3. I am full of energy.

4. I would be happy all the time if I felt as I do now.

Yes

No

5. Things around me seem more pleasing than usual.

Yes $\square$

Yes $\square$

Yes $\square$

Yes $\square$

Yes $\square$

Yes $\square$

Yes $\square$

Yes $\square$

Yes

Yes

Yes $\square$

Yes

Yes

Yes

Yes

Yes $\square$

Yes $\square$

Yes $\square$

Yes $\square$

Yes

Yes $\square$

Yes

Yes $\square$

Yes $\square$

Yes $\square$

Yes $\square$

Yes $\square$

Yes $\square$

No

No

No

No

No

No

No $\square$

No $\square$

No

No

No

No $\square$

No $\square$

No

No

No $\square$

No $\square$

No $\square$

No

No

No

24. I am not as active as usual.

26. I feel drowsy.

No $\square$

No $\square$

27. I am moody.

28. I feel sluggish.

29. I am full of energy.

30. A thrill has gone through me one or more times since I started the test.

32. I feel more clear-headed than dreamy.

Yes

No

No

No

No $\square$

No $\square$

No $\square$

No

33. If you can buy this medication on street, how much are you willing to pay for it?

$\underline{\text { KRW }}$

*The questionnaire was translated into Korean for this study. 